\title{
NOTE SUR LA GESTION AUTOMATIQUE DE LA RÉSERVE POUR SINISTRES EN ASSURANCE AUTOMOBILE*)
}

\author{
par \\ JEAN PfitT \\ Paris \\ Tendances Nouvelles en Automatisme \\ „.. . . La tendance la plus significative qui se dégage \\ ... consiste à doter les systèmes automatiques des \\ facultés d'adaptation qui sont le propre de l'homme. \\ „On constitue ainsi un ensemble doué de mémoire \\ dont la structure se modifie progressivement au fur \\ et à mesure qu'il prend conscience des résultats de \\ ses expériences successives".
}

P. NASLIN

(Automatisme, Nov. 1959)

\section{Avertissement}

Cette note est présentée par un actuaire qui n'a eu que depuis peu à s'intéresser aux branches d'assurances autres que la Vie et seulement du point de vue de la gestion administrative, à l'occasion de la mise en œuvre d'un grand ensemble électronique de traitement de l'information. Il ne faut donc pas s'attendre à trouver ici une théorie sur la détermination des montants à réserver pour les sinistres à régler, encore moins sur la construction des tarifs d'assurance automobile.

Néanmoins, la gestion administrative n'ayant pas pour seul but de recueillir et de classer des données comptables, nous serons bien naturellement conduits à prévoir comment ces données peuvent être liées à des recherches sur ces deux questions fondamentales.

$\mathrm{Si}$ c'est avec un œil neuf et peut-être ingénu que nous nous y engageons, les experts voudront bien nous excuser en considérant que notre propos n'est pas de traiter à fond les problèmes mais

*) Présenté au Colloque de l'Astris ro62 à Juan-les-Pins. 
seulement d'attirer l'attention sur les moyens de les aborder avec les équipements modernes qui permettent:

- un fonctionnement automatique mais souple dans ses modalités,

- des calculs arithmétiques et logiques complexes,

- l'obtention presque instantanée de résultats,

- des analyses très poussées,

- des synthèses multiformes,

tout ce qui est nécessaire, plus encore peut-être aux recherches, qu'à la gestion.

Nos projets se rapportent à l'utilisation d'un grand calculateur électronique à rubans magnétiques mais nous croyons que les idées que nous proposons peuvent bien s'accommoder d'ensembles de moyenne puissance.

\section{ORGANISATION GENERALE}

Dans cet exposé, nous isolons la gestion de la réserve auto sans tenir compte des répercussions nécessaires des sinistres sur les autres parties de l'administration de la Compagnie: (Portefeuille d'Assurances, Quittancement, Comptabilité, etc...) mais, comme on le verra, nous ne préjugeons nullement des liaisons plus ou moins étroites qui peuvent être conçues dans ce domaine.

Pour nous, le problème sous sa forme élémentaire est ainsi délimité: On considère les sinistres déclarés au cours d'une période ou exercice et l'on cherche à déterminer une valeur, disons judicieuse, de la réserve, c'est-à-dire de l'estimation des sinistres restant à régler à la fin de chacun des exercices successifs jusqu'à extinction. Il faut préciser que cette abstraction est employée pour simplifier l'exposé mais qu'il est possible, voire recommandé, de ne faire pratiquement qu'un bloc de tous les sinistres à régler; la discrimination par exercice de survenance est l'affaire du traitement mécanographique.

A leur arrivée, les déclarations sont examinées du point du vue des garanties et une première estimation est effectuée, dans des conditions que nous préciserons ultérieurement.

C'est alors qu'elles sont enregistrées mécanographiquement pour donner naissance au "fichier" des sinistres de tel exercice, quel qu'en soit le support, cartes ou ruban magnétique. 
Par la suite toute information recueillie sera enregistrée et donnera lieu, suivant les cas, à une addition ou une modification, de l'information primitive; il en sera ainsi notamment des règlements effectués et des déclarations devenues sans objet que l'on éliminera si l'on veut des calculs, le moment venu.

Ce n'est que lorsque, pratiquement, le sort de toutes les déclarations d'un exercice aura été réglé que le fichier en question pourra être relégué.

On disposera ainsi intégralement de toute la documentation relative à un exercice de survenance.

Ces opérations sont, mécanographiquement très faciles: il suffit d'une carte ou bande perforée comportant pour chaque "mouvement" un indice de repérage correspondant au classement du fichier (numéro de sinistre et exercice de survenance par exemple, joint à un second élément de sécurité: Nom ou Numéro de police ....), un code d'opération à effectuer, le contenu de l'information à ajouter ou modifier.

La mise à jour du fichier sera faite à des périodes aussi rapprochées qu'il peut être utile.

Nous reviendrons sur ces questions d'enregistrement des informations car nous sommes convaincus qu'un fichier de ce genre bien tenu et auquel aucune donnée n'échappe, non seulement est suffisant, mais encore doit être unique pour assurer tous les besoins de gestion comptable, de statistiques et de recherches d'une Compagnie relativement aux sinistres déclarés, en liaison avec les autres secteurs administratifs.

C'est ainsi qu'on peut imaginer sans peine qu'un rapprochement des déclarations avec le fichier général des polices pourrait servir à la vérification automatique des garanties en même temps qu'à un enregistrement historique de rappel par police.

Les accusés de réception, demandes d'expertises, exercice des recours etc..., et plus tard les contrôles de règlements peuvent s'organiser avec les opérations d'entrée, de mise à jour et d'extraction d'informations du fichier des sinistres, de même que les transmissions d'informations aux organismes d'études en commun.

Mais nous n'entrerons pas dans plus de détails, nous bornant à la question de la ,réserve". 


\section{Premiere estimation et PARAMEtres D'Estimation}

Le commencement de tout ce qui a rapport à la réserve auto est la première estimation. Peu importe la règle; que ce soit à une valeur moyenne en distinguant si l'on veut les cas qui sont supposés devoir être graves, que ce soit par un examen minutieux de chaque déclaration, ou tout autre méthode, simple ou composée.

On pourrait même envisager de relever seulement les états de faits pouvant conduire, après des regroupements et à travers des formules ou des tables de correspondance, à des évaluations.

Quelque soit le système, nous proposons d'introduire en outre, des éléments que nous nommerons des ,,paramètres d'estimation", affectés à chaque déclaration.

Nous admettons en effet, que les premières estimations sont révisables dans l'avenir, non seulement en raison de nouvelles informations qui șont particulières à chaque sinistre, comme nous l'avons déjà dit, mais aussi en fonction de considérations d'ordre général.

La nature et la base des paramètres de cette espèce étant fixées par exemple au début d'un exercice, nous supposons que chaque sinistre peut, au moment de la première estimation être coté, comme très sensible, sensible ou indépendant de la variation de l'indice correspondant à chacun de ces paramètres.

Ainsi il sera facile, à toute époque de faire subir aux évaluations une modification générale mais nuancée, ou même seulement d'étudier les conséquences sur la réserve d'un événement survenu ou attendu touchant à la nature des paramètres introduits au départ.

Nous ne nous laisserons pas aller jusqu'à rechercher ou conseiller le choix de tels paramètres, laissant ce soin à de plus expérimentés; nous n'en donnerons que deux exemples notoires, pour fixer les idées.

\section{Facteur monétaire}

On peut affecter la première évaluation, soit d'une base arbitraire, soit d'un indice économique, en prenant la précaution élémentaire d'assurer la concordance entre l'application de la règle et l'indice, de telle sorte que l'ensemble "Evaluation-Indice" permette de 
rendre tous les sinistres comparables, non seulement à l'intérieur d'un exercice mais pour plusieurs exercices consécutifs.

On pourra différencier les dommages ou fractions de dommages en les faisant dépendre de divers indices selon leur nature: indices de prix, de salaires, etc., en utilisant des codes rapportés aux éléments d'évaluation.

Noter que, mécanographiquement, il suffit d'un code de nature d'indice, lequel rapproché, par exemple, d'une date, fera découvrir le montant de l'indice par recherche automatique dans une table préalablement enregistrée en machine.

\section{Facteur juridique}

On a souvent connaissance par avance d'une évolution possible de la jurisprudence dans des questions plus ou moins définitivement tranchées.

On pourra introduire un paramètre avec des cotes différentes suivant que l'on craint des conséquences importantes pour certains sinistres, faibles ou nulles pour d'autres.

\section{Montant de la réserve}

Bien entendu les natures des divers paramètres doivent être nettement distinctes, de telle sorte que la variation de l'un ne produise pas une variation d'un autre.

Tout ceci n'est donné qu'à titre d'exemples, mais il faut bien comprendre que l'on pourrait, dès la fin de l'exercice de survenance, „SIMULER", pour employer une expression de la Recherche Opérationnelle, ce qui se passerait dans les cinq ou six exercices à venir, en fonction des lois hypothétiques nécessaires (cadence des réglements, évolution monétaire, jurisprudence...) et pour diverses valeurs des paramètres correspondants.

Ceci aiderait sans doute à arrêter le montant de la réserve avec la meilleure connaissance possible des choses.

\section{ANALYSE DES RESUlTATS PARAMETRES D'OBSERVATION}

Dès la fin du premier exercice, et ensuite, pour les exercices ultérieurs, les informations modifiant le premier enregistrement 


\section{SCHEMA FONCTIONNEL}

Informations externes

Ouverture du dossier
Estimation initiale
paramétrée

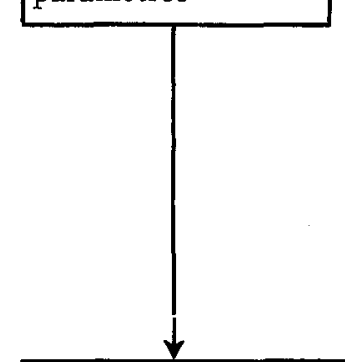

- Enregistrement mécanographique

- Création de documents administratifs et comptables correspondants

- Statistique des Mouvements

Informations sur dossier ouvert

Modification :

- données initiales

Règlement:

- total

- partiel

- sans suite -_estimations initiales
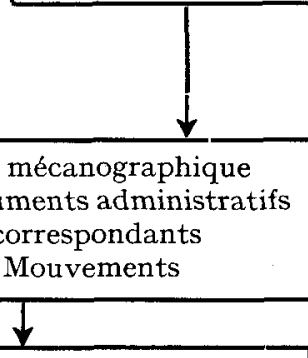

Mise à jour du Fichier des Sinistres

- Premier calcul de la réserve

- Comparaisons avec les estimations antérieures

- Calcul des paramètres d'observation

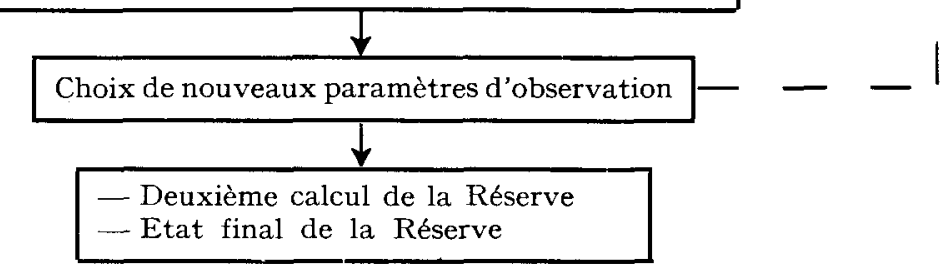

Informations internes

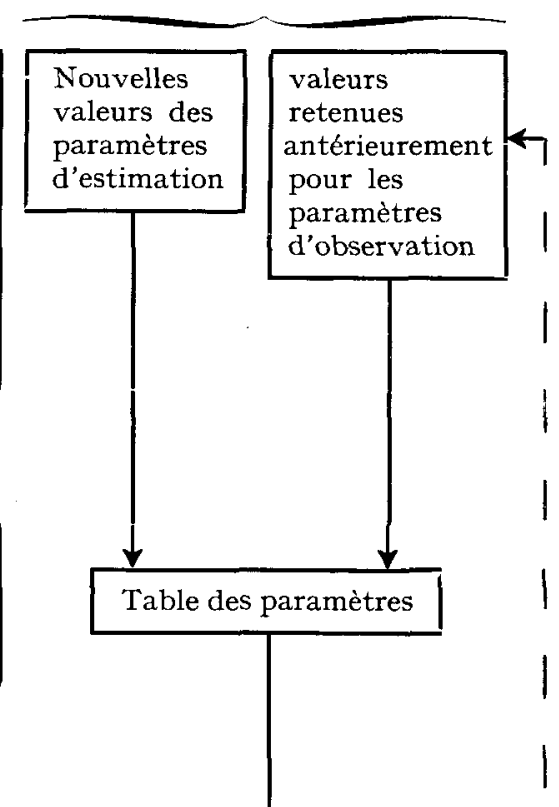

(essentiellement les réglements effectués), pourront être rapidement analysées en fonction des paramètres d'estimation qui avaient été définis et notés. Comme les événements survenus dans l'intervalle qui influent sur ces paramètres sont alors connus on aura pu décider s'il y a lieu de donner de nouvelles valeurs à leurs bases.

C'est ainsi qu'en effectuant des comparaisons, soit globales, soit 
fragmentaires entre les premières évaluations corrigées par les paramètres d'évaluation et les réglements effectifs, on pourra se faire une opinion sur la qualité des paramètres d'estimation et des bases utilisées.

On surveillera, bien entendu, dans le temps la cadence des réglements en fonction de leur importance et tous autres éléments d'appréciation pouvant être extraits des données.

Les différences, globales ou particulières à des groupements étudiés, pourront, selon les interprétations qu'elles auront inspirées, donner naissance à un ou de nouveaux coefficients que nous appellerons ,,paramètres d'obscrvation".

Ceux-ci pourront être affectés à un ou plusieurs exercices, à des catégories déterminées ou même à certains dossiers isolés.

On pourra ainsi calculer ou étudier, à la fois d'après les paramètres d'estimation et d'observation, des corrections aux premières évaluations des sinistres non encore réglés, en vue de donner si on le juge utile en vertu de considérations financières et de sécurité, une nouvelle valeur très élaborée à la réserve à la fin de l'exercice en cause.

C'est cet aspect expérimental d'élaboration continue qui permet d'attribuer un caractère d'automaticité, à la gestion de la réserve.

\section{EXPLOITATION DU FICHIER - RECHERCHES}

L'un des principaux attraits d'une telle organisation est la possibilité d'examiner rapidement les effets de la variation de tel ou tel facteur ou de plusieurs facteurs conjugués sur les évaluations de sinistres non encore réglés, à n'importe quel moment, même en cours d'exercice, sur le fichier mis à jour.

Il est en principe nécessaire que l'intervention des facteurs ait été prévue dès le premier enregistrement, ou, au moins, que les éléments dont on peut supposer qu'ils pourront avoir une influence sur le montant des réglements aient été recueillis.

A ce propos, nous pensons qu'il n'y a pas lieu de lésiner sur le volume des renseignements à enregistrer à l'occasion des déclarations de sinistres. En effet, si la quantité d'informations constitue une lourde charge lorsqu'il s'agit du portefeuille des assurances en cours, il n'en est plus tout à fait de même pour les sinistres qui sont infiniment moins nombreux. 
En outre, il est peut-être moins difficile alors d'exiger certaines données complètes et précises, plutôt qu'à la souscription, sans parler de celles qui n'existent ou ne sont valables que par leur actualité au moment de l'accident.

Nous ne croyons pas non plus qu'il y ait à redouter un trop lourd fardeau pour le travail matériel que constitue l'enregistrement. Il est possible de combiner une fiche- questionnaire sur laquelle le vérificateur n'ait qu'à porter des croix, qui fixent elles-mêmes la codification, en vue d'une perforation immédiate des cartes mécanographiques; c'est une organisation devenue coutumière. L'allégement des travaux de gestion permet de soigner davantage l'arrivée des informations: il s'agit de tirer le meilleur parti de toutes les données d'analyse dont on peut disposer en vue de recherches.

Ceci conduit naturellement à discuter de la qualité des informations.

Pour le mécanographe, il suffit qu'elles soient autant que possible codées en tout cas définies sans ambiguïté et qu'elles soient sûres c'est-à-dire fournies ou transcrites sans erreur. C'est un aspect extérieur de l'information.

Pour le gestionnaire c'est leur signification qui est essentielle. Pour le statisticien c'est encore autre chose: elles doivent correspondre à des idées, pouvant servir de point de départ à des recherches, mais le malheur est que l'on ne sait pas toujours d'avance comment seront plus tard orientées les recherches et ce dont on aura besoin.

C'est pourquoi nous tendrions volontiers à noter tout ce qui est notable c'est-à-dire ce que l'on peut obtenir de bien défini, d'indiscutable et il est probable que cela limitera la quantité des enregistrements. D'ailleurs il n'est pas exclu d'essayer, notamment par des sondages, de se rendre compte si certaines données méritent d'être enregistrées pour en suivre ultérieurement l'influence complète ou l'évolution.

Le fichier des sinistres ainsi conçu permettra de nombreuses études en vue de vérifier des hypothèses, de surveiller les résultats de la politique propre à la Compagnie, la stabilité des coûts moyens, des taux de responsabilité, des cadences de réglements, des causes d'accidents pour servir à la prévention etc, de rechercher de nou- 
velles lois ou de chiffrer les conséquences de nouveaux concepts avancés.

Certes les recherches effectuées sur les seuls sinistres ne permettent pas de remonter aux ensembles de risques pouvant servir de bases à la tarification; c'est inversement qu'on procède, on cherchant à justifier expérimentalement les groupements répondant à des conceptions logiques a priori. Mais on peut penser que des études de sinistres menées sous plusieurs points de vue différents pourraient guider dans le choix des définitions.

L'ensemble des fichiers de ce genre qui contiennent toutes les données relatives aux sinistres permettrait avec un système de repérage adapté de résoudre les questions qui se posent à propos du bonus, tant statistiques que de détermination et de distribution ou même simplement de contrôler le ,passé-sinistres” avec les déclarations.

Cette idée de repérage qui peut être étendue aux conducteurs, témoins, victimes par un procédé analogue à celui du fichier des refus en assurances Vie suggère une possibilité de déceler des fraudes qui ne sont actuellement découvertes que par hasard et après de nombreuses années. L'expérience nous a enseigné que des systèmes de comparaisons automatiques portant sur des compatibilités ou des vraisemblances entre des données, même au besoin, grâce à des redondances organisées, permettent, en signalant les anomalies, des contrôles très étendus et d'une parfaite efficacité.

Nous ne pouvons pas omettre de considérer le point de vue des Autorités de Contrôle.

Sur le plan des principes, et puisqu'on ne dispose guère de critères objectifs permettant d'optimaliser le montant de la réserve, nous pensons qu'il faut se contenter d'un accord sur le choix et la valeur des paramètres à mettre en jeu à partir d'une première règle acceptée. Quant aux moyens de vérifier l'application des règles et l'exactitude des calculs, ils ne posent pas de problèmes redoutables: le fichier permet de sortir au ligne à ligne si c'est nécessaire tous les éléments de calcul et le montant de la réserve pour chaque sinistre; par conséquent on pourra aussi bien obtenir tous groupements et opérer tous calculs collectifs ou approchés permis par les formules. 
Le projet dont nous avons décrit les grandes lignes montre comment on peut profiter de la mécanisation pour chercher à résoudre les problèmes de gestion et à poursuivre les études sur la réserve auto. Il pourra paraître trop ambitieux à certains mais rien n'empêche d'en limiter l'application. Cependant, il se posera toujours de nouveaux problèmes et nous espérons avoir tracé un cadre assez large pour qu'ils puissent y être insérés. 